\title{
Ohm's Law Refutes Current Version of the Special Theory of Relativity
}

\author{
Alexander Alexandrovich Antonov \\ Research Centre of Information Technologies “TELAN Electronics”, Kiev, Ukraine \\ Email: telan@bk.ru
}

How to cite this paper: Antonov, A.A. (2016) Ohm's Law Refutes Current Version of the Special Theory of Relativity. Journai of Modern Physics, 7, 2299-2313. http://dx.doi.org/10.4236/jmp.2016.716198

Received: November 17, 2016

Accepted: December 12, 2016

Published: December 15, 2016

Copyright $\odot 2016$ by author and Scientific Research Publishing Inc. This work is licensed under the Creative Commons Attribution International License (CC BY 4.0).

http://creativecommons.org/licenses/by/4.0/ (c) (i) Open Access

\begin{abstract}
It is shown that Ohm's law is not only the main electrical engineering law, but also a generally scientific worldview law of the extreme significance, as in the interpretation of Steinmetz it proves physical reality of imaginary numbers theoretically and experimentally in the most indisputable way. Thus, it refutes the principle of light speed non-exceedance, which is fundamental in the special theory of relativity. Moreover, unlike the MINOS and OPERA experiments recognized by physical community as not enough reliable, which were conducted for the same purpose, alternative experiments were performed during the research of oscillation processes in linear electric circuits. Therefore, they are absolutely reliable and conclusive as can be repeated and verified in any electrical engineering laboratory. The principle of physical reality of imaginary numbers proven by the electrical engineering experiments is generally scientific, since mathematics is the universal language of the exact sciences. Therefore, all scientific theories and hypotheses in quantum mechanics, relativity theory, geophysics, cosmology, optics, radio electronics and other sciences should be adjusted accordingly, given the principle of physical reality of imaginary numbers. There is an example of how this can be done in the special theory of relativity and astrophysics. This approach allowed explanation of dark matter and dark energy, which correspond to the invisible parallel universes existing in extra dimensions.
\end{abstract}

\section{Keywords}

Ohm's Law, Imaginary Numbers, Special Theory of Relativity, Dark Matter, Dark Energy, Multiverse

\section{Introduction}

In 1826, after nine years of experimental research, the great German physicist Georg 
Simon Ohm (1787-1854) published his article [1] about the law that bears his name today. To understand how uneasy it was, it's enough to recall that there were no electric metering devices at that time. In 1827, he published a book [2], which laid the foundations of the theory of linear electric circuits where he used his own terms "electromotive force", "voltage drop", "current intensity", "resistance" and "conductance". He even explains how to describe electric circuits in which both conductors and current sources are interconnected either in series or in parallel.

However, Ohm's contemporaries were more than cool to his discoveries, because at that time it was commonly supposed that the truth in science should be learned theoretically, whereas experience could not (and even should not!) be taken into account. And according to Ohm's critics (including top-ranking) his works smelled rather of sweat than of "supreme harmony". In this regard, the famous Russian physicist Alexander Grigorevich Stoletov (1839-1896) wrote: “...physics especially tempted natural philosophers. What a favorable theme were electrical phenomena for the most riotous imaginations... Attractive and vague deductions were in the foreground: hard work of experimenter and exact mathematical analysis were not honored; they seemed superfluous and harmful in the study of nature...".

In 1828, Ohm was even fired where he worked by personal order of Minister of Education for publishing his physical discoveries. A senior official believed that the use of mathematics in physics was unacceptable. Only after French physicist Claude Servais Mathias Pouillet (1791-1868) obtained the same experimental results in 1831-1837, Ohm's law was recognized by scientific community.

In 1897, the eminent American engineer and inventor Charles Proteus Steinmetz (1865-1923) suggested his own interpretation of Ohm's law [3], as applied to AC-circuits. In accordance with this interpretation, electric LCR-circuits are peculiar to resistors, capacitors and inductors. Unlike resistors, resistance of which is measured by real numbers, reactance of capacitors and inductors is measured by heteropolar imaginary numbers.

Currently, Ohm's law is studied in the school course of physics, and therefore it is known to all educated people. However, its great significance to all exact sciences has not been still realized. Even in physics.

This feature of Ohm's law is covered in the article.

\section{Physical Reality of Imaginary Numbers}

The main advantage of Ohm's law in the interpretation of Steinmetz turned out to be the fact that in addition to capability to calculate electric circuits, it allows convincing and indisputable proof of physical reality of imaginary numbers, which have been fiscovered over five hundred years ago by Scipione del Ferro (1465-1525), Niccolò Fontana Tartaglia (1499-1557), Gerolamo Cardano (1501-1576), Lodovico Ferrari (1522-1565) and Rafael Bombelli (1526-1572) [4].

Mathematics and other exact sciences have always developed simultaneously, including in the process of emergence of new types of numbers and better understanding 
of their sense. Thus, integers appeared first. Then, there were fractions, positive and negative numbers, etc. But when the process concerned the imaginary, complex and hypercomplex numbers, they ceased being understood. Works of AbrahamdeMoivre (1667-1754), Leonhard Euler (1707-1783), Jean Le Rond D'Alembert (1717-1783), Caspar Wessel (1745-1818), Pierre-Simonde Laplace (1749-1827), Jean-Robert Argand (1768-1822), Johann Carl Friedrich Gauss (1777-1856), Augustin Louis Cauchy (17891857), William Rowan Hamilton (1805-1865), Pierre Alphonse Laurent (1813-1854), Karl Theodor Wilhelm Weierstrass (1815-1897), Georg Friedrich Bernhard Riemann (1826-1866), Oliver Heaviside (1850-1925), Jan Mikusiński (1913-1987) and of many other outstanding mathematicians contributed to the development of a perfect complex variable theory [4]. However, it hasn't led to any understanding of physical essence of imaginary numbers.

Therefore, due to inability to explain imaginary values of mass, time and other physical quantities appearing in relativistic formulas at superluminal speeds within the special theory of relativity (STR) [5] [6] [7], developed at the beginning of the 20th century by Joseph Larmor (1857-1942), Nobel Prize winner Hendrik Antoon Lorentz (18531928), Jules Henri Poincaré (1854-1912), Nobel Prize winner Albert Einstein (18791955 ) and other prominent scientists, its authors had actually to postulate (as it hadn't ever been proved) the principle of light speed non-exceedance [8].

The principle seemed to be substantiated quite conclusively. Since the value of relativistic mass $m$ depends on the velocity $v$ in the Lorentz-Einstein formula:

$$
m=\frac{m_{0}}{\sqrt{1-(v / c)^{2}}}
$$

where $m_{0}$ is the rest mass of a moving entity (e.g. elementary particle);

$m$ is the relativistic mass of a moving entity;

$v$ is the velocity of a moving physical entity;

$C$ is the speed of light.

Mass $m$ takes, at near-light speed, values approaching to infinitely large real values, i.e., $\lim _{v \rightarrow c} m(v)=\infty$, given that $v<c$. Therefore, overcoming of light speed barrier requires infinite energy, which is physically impossible. So, impossible is the overcoming. It can be concluded that there is nothing behind the barrier and we live in a monoverse. Consequently, it would make no sense to explain the situation, when $v<c$.

However, such substantiation is refuted even by simple household arguments. Indeed, inability to overcome a barrier dividing two adjacent rooms in our house, which is a wall in this case, does not mean that the next room does not exist or that there is no possibility to get into it through the door. The universe, which is arranged much more complicated than our house, can actually have its possibilities to overcome the light speed barrier. And it really does, as shown below.

Therefore, such a simple solution of a complex physical-mathematical problem offered by the authors of the STR did not seem convincing to everyone. At the beginning of the twenty-first century very complex MINOS [9] and OPERA [10] experiments were conducted at the American Tevatron Collider and he European Large Hadron Collider, 
accordingly. They aimed to detect superluminal neutrinos and thus prove physical reality of imaginary numbers. However, physical community considered the experiments unreliable and denied them by the ICARUS experiment [11]. Or, to be more precise, ICARUS experiment denied only the refutation of the principle of light speed non-exceedance alleged by the MINOS and OPERA experiments, but didn't proved the validity of this principle. It even didn't aim to.

However, as according to the author of the "open society" concept Karl Raimund Popper [12]: "The struggle of opinions in scientific theories is inevitable and is a necessary prerequisite for the development of science". Therefore, attempts to refute the principle of light speed non-exceedance in the STR were not limited to the OPERA and MINOS experiments. Other experiments [13]-[19], using resonance and transient oscillation processes in linear electric circuits, that did prove physical reality of imaginary numbers, were conducted almost simultaneously with MINOS and OPERA experiments. Moreover, in contrast to the MINOS and OPERA physical experiments, the electrical engineering experiments turned out to be absolutely reliable, as they could be repeated and verified in any electrical and radio electronic laboratory.

Since mathematics is the common language of all exact sciences, the principle of physical reality of imaginary numbers proved by electrical engineering experiments is valid for all other exact sciences. Consequently, all the theories and hypotheses of quantum mechanics, relativity theory, optics, radio electronics, geophysics and other sciences should be adjusted accordingly.

Whereas dozens of scientific publications were devoted to searching for ways of refutation of the OPERA experiment (between the dates of publication of OPERA and ICARUS experimental results) in a half-year period, alternative electrical engineering experiments have not been commented in at least one article over the past few years. Some of the publications criticizing the OPERA experiment even claimed that the principle of light speed non-exceedance in the STR was so important that if the OPERA experiment refuted it, its authors would have deserved the Nobel Prize for such a scientific achievement. However, the principle turned out to be refuted and the authors of the publications immediately lost interest in this issue.

Therefore, one cannot but suppose that the refuters of the MINOS and OPERA experiments needed confirmation of the alleged irrefutability of the principle of light speed non-exceedance and thus preservation of the belief in accuracy of the existing STR version rather than scientific truth. However, unlike the results of the MINOS and OPERA experiments, the results of the alternative electrical engineering experiments turned out to be quite reliable and irrefutable. Consequently, they did refute the existing version of the SRT.

\section{The Proof of the Principle of Physical Reality of Imaginary Numbers Using the Ohm's Law in the Interpretation of Steinmetz}

The most interesting thing is that the current version of the STR has been actually re- 
futed by Charles Proteus Steinmetz by Albert Einstein's famous publication [20] in 1897 [3], i.e., before the date of its development in 1905.

Indeed, according to the Steinmetz's interpretation of the Ohm's law not only resistors, but also inductors and capacitors have electric impedance. Besides, impedance of capacitors and inductors is measured by imaginary numbers, the value of which depends on the frequency of sinusoidal voltage applied to the electric LCR-, LC-, RC- and RL-circuits. However, under the dominant influence of STR, denying physical reality of imaginary numbers, imaginary impedance of capacitors and inductors is actually believed to be imaginary, i.e., physically non-existent.

The fallacy of this opinion is easily verified experimentally by changing the frequency of sinusoidal voltage applied to any electric LCR-, LC-, RC- or RL-circuit and measuring the value of electric current flowing through the circuit. All engineers have known for over a century that the electric current is changed in such experiments. This is possible only if frequency-dependent impedance of capacitors and inductors really exists. Moreover, instruments for measuring frequency characteristics of electric circuits confirming such changes have been created many decades ago and still widely used in engineering practice. Consequently, imaginary electric impedance of capacitors and inductors is physically existent [21] [22] [23]. There are also instruments widely used for measurement of supposedly imaginary impedance.

Finally, physical reality of impedance of capacitors and inductors is also confirmed by the existence of resonance phenomenon [24] in electric circuits, discovered by Galileo di Vincento Bonaiutide' Galilei in 1602. If there were no resonance in electric circuits, such sciences as radio engineering, telecommunications, radiolocation and many others wouldn't exist.

All this indisputably confirms physical reality of imaginary numbers. As well as existence of the relevant instruments and measurements made using them confirms the existence of ultra- and infrasound, ultraviolet and infrared radiation, magnetic and gravitational fields, X-rays and radioactivity, and almost everything else that we know about the world around us.

In the early twentieth century, authors of the STR did not know that. However, supporters of the existing version of the STR keep not knowing that even now, as they are convinced of the validity of the principle of light speed non-exceedance.

\section{Physical Essence of Imaginary Numbers in the Special Theory of Relativity}

For a complete understanding of the physical essence of imaginary numbers $a i$, where $a$ is the real number, and $i=\sqrt{-1}$ is the imaginary unit, proving their actual physical existence is, however, insufficient. It should be clear what they represent in each case. For example, it was explained above that $+10 i \mathrm{ohms}$ and $-20 i \mathrm{ohms}$ are electric impedances of an inductor and capacitor. This is well illustrated by vector diagrams.

However, what, for example, does $10 i$ gr or $20 i \mathrm{sec}$ mean? This question is more difficult, but we try to give an answer. 
For this purpose let's use the formula (1). It follows from the formula that relativistic mass of the moving body takes real values for $v<c$ and imaginary values for $v>c$. It has been proved above that concrete imaginary numbers are physically existent. Therefore, imaginary relativistic mass of, let us say, tachyons [25], [26] is as physically real as real relativistic mass of tardyons. Other relativistic formulas show that relativistic time, length and momentum are also real and imaginary. Therefore, the statement of the STR that there is nothing physically real behind the light speed barrier and the principle of light speed non-exceedance justifying this statement are incorrect [27].

However, if the imaginary physical quantities are real, and physical bodies to which they belong exist in the universe other than ours (as in our universe the situation $v>c$ is impossible), these physical bodies move in another universe which we, for certainty, would call a tachyon. For similar reasons, our universe would be called a tardyon. We cannot see tachyon universe from our tardyon universe, because in view of the condition $v>C$ it is outside the event horizon.

Consequently, contrary to the statement of the STR about existence of monoverse, we still live in a Multiverse. It is invisible, so let it be referred to as the hidden Multiverse [28].

The answer to the question risen above is that the $10 i \mathrm{gr}$ and $20 i \mathrm{sec}$ in our tardyon universe are $10 \mathrm{gr}$ and $20 \mathrm{sec}$ in the tachyon universe. It follows that the values of physical entities in the adjacent parallel universe correspond to imaginary numbers in astrophysics.

Tardyon and tachyon universes of the hidden Multiverse should be indistinguishable in their manifestations for their inhabitants, i.e., according to the first postulate of the STR tachyon universe has the same physical, chemical, biological and other laws as in our universe, as well as it has its own life and its own intelligent inhabitants. However, relativistic formulas, including the formula (1), do not meet this condition. Therefore, the formula (1) shall be amended as follows:

$$
m=\frac{(i)^{q} m_{0}}{\sqrt{1-(v / c-q)^{2}}}=\frac{(i)^{q} m_{0}}{\sqrt{1-(w / c)^{2}}}
$$

where $q=[v / c]$ is the discreet "floor" function of argument $v / c$;

$w=v-q c$ is the local velocity for each universe, which can take values only in the range $0 \leq w \leq c$;

$v$ is the velocity measured from our universe, which can therefore be called space velocity;

$c$ is the speed of light;

$i=\sqrt{-1}$ is the imaginary unit.

Other relativistic formulas can be adjusted in a similar manner.

\section{Physical Essence of Imaginary Numbers in Astrophysics}

\subsection{Multiverse, Parallel Universes, Extra Dimensions}

It follows from the formula (2) that tardyon universe corresponds to the value $q=0$ 
(as $i^{0}=1$ ), and tachyon universe corresponds to the value $q=1$ (as $i^{1}=i$ ). However, the formulas (3) and (4) do not imply that the value $q$ can be greater than one. It is shown below that there are actually more than twenty parallel universes in the hidden Multiverse (since they never intersect despite their infinity).

Thus, tardyon antiverse would correspond to the value $q=2$ (as $i^{2}=-1$ ), tachyon antiverse would correspond to the value $q=3$ (as $i^{3}=-i$ ), another tardyon universe would correspond to the value $q=4$ (as $i^{4}=1$ ), another tachyon universe would correspond to the value $q=5$ (as $\left.i^{5}=i\right)$ and so on.

That is, the Multiverse has a structure of either a closed (as in Figure 1) or an open

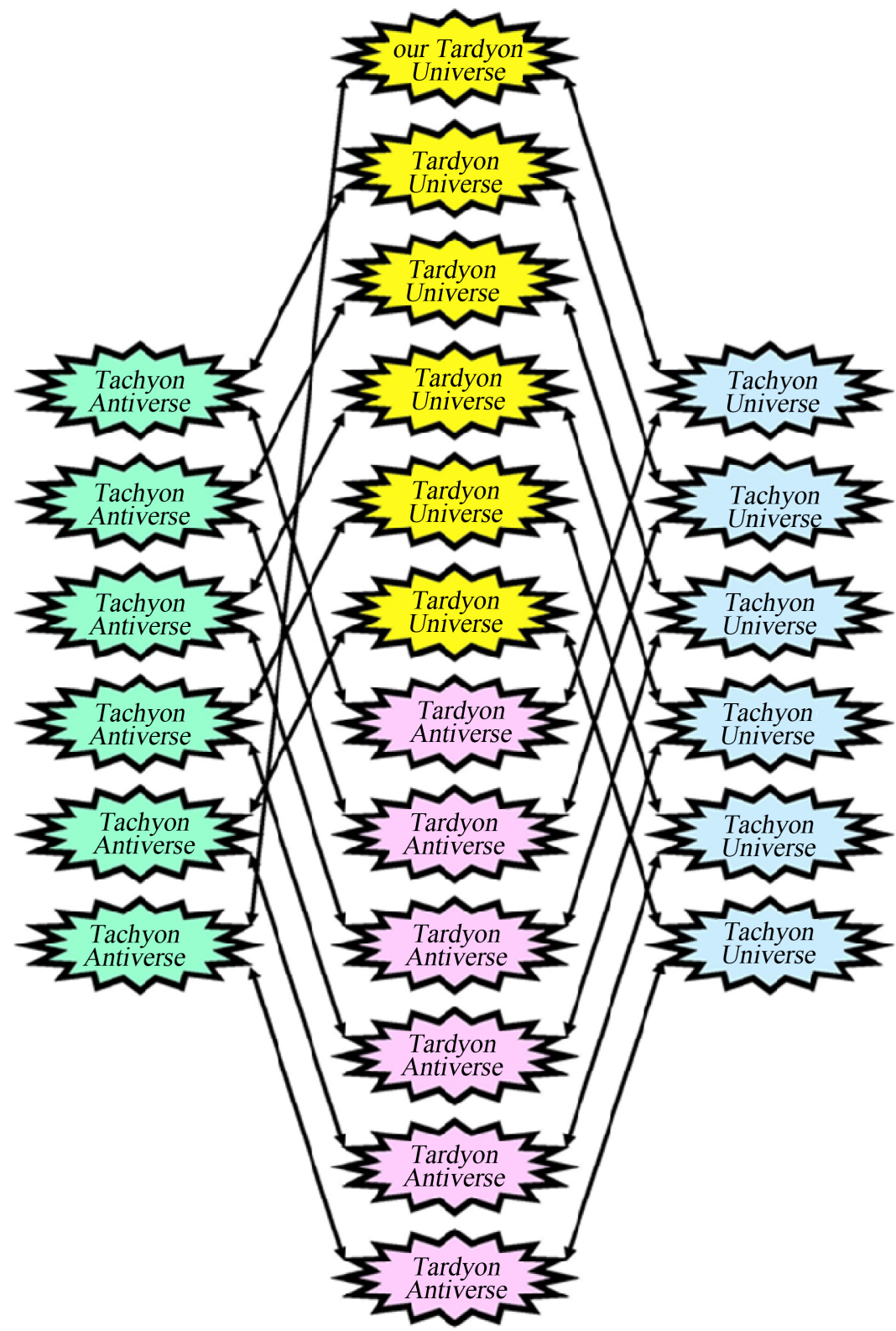

Figure 1. Probable structure of the hidden Multiverse corresponding to the principle of physical reality of complex numbers. 
screw collar. Stephen William Hawking apparently meant that when said: "Imaginary time is a new dimension, at right-angles to ordinary, real time".

The value $q$ can be regarded in the above structure as an extra spatial dimension (like numbers of apartments in apartment buildings, each of which has its three spatial dimensions).

Besides, parallel universes are continuously drifting in this four-dimensional space and occasionally or permanently touch each other (for example, as people in a crowd) or even slightly penetrate into each other in some spots (which are quite numerous, including those on Earth). Transition zones which in literature are also referred to as portals or star gates emerge in the places of such local interpenetrations of universes. Micro objects ranging from elementary particles to living inhabitants and their vehicles can transit from one parallel universe to another. Exception is stars and galaxies, since parallel universes could lose their stability. Portals on the Earth are so-called anomalous zones [29]. Portals between parallel universes are denoted by single bidirectional arrows.

\subsection{Phenomenon of Dark Matter and Dark Energy}

However, in addition to the observed and, therefore, most understandable research objects (planets, stars, galaxies, etc.) in astrophysics, there are less understandable objects (such as black holes), and even completely obscure objects of research (dark matter and dark energy). Dark matter and dark energy [30], [31] are of the greatest interest among them. First of all, it is because mass-energy of dark matter and dark energy is more than twenty times greater than mass-energy of the entire visible universe. The other reason is that dark matter and dark energy is completely invisible in all ranges of electromagnetic radiation. Finally, dark matter and dark energy are of the greatest interest because no chemical element known to us has been found in their structure, which even poses risk to the modern understanding of the term "matter" and sets the science millennia back. Therefore, these objects of study have been called dark.

Taking into account all these features of dark matter and dark energy the problem of their explanation, to which no one could even come close in the last decade, becomes ideologically important not only for astrophysics but also for science as such. Stephen William Hawking wrote: "The missing link in cosmology is the nature of dark matter and dark energy".

However, the problem of explaining the phenomenon of dark matter and dark energy turned out to be inexplicable, because under the dominant influence of the STR the statement of explanation task was formulated incorrectly. Its explanation has been required to be found exactly within the monoverse hypothesis corresponding to the current version of the STR, which, as shown above, is wrong. Therefore, it hasn't been and it wouldn't be found under such conditions. Albert Einstein stated in this regard: "Insanity: doing the same thing over and over again and expecting different results".

If statement of the problem is changed and explanation of the phenomenon of dark matter and dark energy is found within the Multiverse hypothesis, it would become 
apparent [32]-[39]:

- Invisible dark matter and dark energy are invisible parallel universes of the hidden Multiverse other thanours;

- Dark matter is parallel universes of the hidden Multiverse adjacent to our universe.

- Dark energy is parallel universes of the hidden Multiverse other than our and adjacent universes;

- Chemical composition of parallel universes of the hidden Multiverse, except ours, cannot be determined because their content is not available for analysis by our earthly tools.

Explanations provided seem quite unusual. But Sir Isaac Newton believed: "No great discovery was ever made without a bold guess".

\subsection{Quaternion Structure of the Hidden Multiverse}

Data obtained from the WMAP [40] and Plank spacecrafts [41] are extremely important for determining the structure of the hidden Multiverse, because they allow us to calculate its basic parameters.

According to the data, the total mass-energy of the whole hidden Multiverse consists of $4.9 \%$ baryonic matter (earlier WMAP estimate-4.6\%), 26.8\% dark matter (according to WMAP-22.4\%) and 68.3\% dark energy (according to WMAP-73\%). Therefore, believing that mass-energy of all parallel universes is equal (as a result of its averaging through portals), it can be concluded that:

- Mass-energy of our tardyon universe is $4.9 \%$ of the mass-energy of the hidden Multiverse according to Planck data;

- The hidden Multiverse consists of $100 \% / 4.9 \%=20.4$ universes according to Planck data (or of $100 \% / 4.6 \%=21.7$ universes according to earlier WMAP estimate);

- Dark matter consists of $26.8 \% / 4.9 \%=5.5$ universes according to Planck data (or of $22.4 \% / 4.6 \%=4.9$ universes according to earlier WMAP estimate);

- Dark energy consists of $68.3 \% / 4.9 \%=13.9$ universes according to Planck data (or of $73.9 \% / 4.6 \%=15.9$ universes according to earlier WMAP estimate);

Thus, according to the data obtained from WMAP and Plank spacecrafts the hidden Multiverse contains twenty to twenty-two parallel universes, one of which is our universe, five or six parallel universes are its neighbors, and the other parallel universes are still further from us.

However... the results are inconsistent with the structure of the hidden Multiverse shown in Figure 1 as there are actually five or six parallel universes adjacent to tardyon universes rather than two ones including a tachyon universe and a tachyon antiverse. Nevertheless, this does not mean that the principle of physical reality of imaginary numbers is inapplicable in astrophysics. In fact, only the simplest case of its use in the form of complex numbers having one imaginary unit $i$ turned out to be inapplicable. And since our tardyon universe is adjacent only to five or six parallel universes, the number of extra dimensions should be increased to three and therefore, one more adjusted Lorentz-Einstein formula should contain three imaginary units $i_{1}, i_{2}, i_{3}$ 


$$
m=\frac{m_{0}\left(i_{1}\right)^{q}\left(i_{2}\right)^{r}\left(i_{3}\right)^{s}}{\sqrt{1-[v / c-(q+r+s)]^{2}}}=\frac{m_{0}\left(i_{1}\right)^{q}\left(i_{2}\right)^{r}\left(i_{3}\right)^{s}}{\sqrt{1-(w / c)^{2}}}
$$

Formula (3) includes similar designations as in the formula (2).

Therefore, the hidden Multiverse has the structure [42] that confirms physical reality of quaternions [43], containing three imaginary units $i_{1}, i_{2}, i_{3}$ interconnected by the following relations:

$$
\begin{gathered}
i_{1}^{2}=i_{2}^{2}=i_{3}^{2}=-1 \\
i_{1} i_{2} i_{3}=i_{2} i_{3} i_{1}=i_{3} i_{1} i_{2}=-1 \\
i_{1} i_{3} i_{2}=i_{2} i_{1} i_{3}=i_{3} i_{2} i_{1}=1
\end{gathered}
$$

Figure 2 presents a quaternion structure of the hidden Multiverse in the form of a screw collar. It contains bidirectional portals (same as in Figure 1) corresponding to the relation (4a) denoted by single bidirectional arrows and unidirectional portals corresponding to the relations (4a) (4b), denoted by single unidirectional arrows. Our tardyon universe is both conditional beginning and end of reference of parallel universes in the closed structure of the hidden Multiverse.

However, this structure also does not correspond to the parameters of the hidden Multiverse, calculated according to the WMAP and Planck data, as it contains twenty-four parallel universes rather than twenty or twenty-two. It actually could be turned into the structure corresponding to the WMAP and Planck data by excluding two or four universes from it. In this case it would have edges, and the most important of all it raises the question of what is hidden behind the edges of the Multiverse. There are no experimental data that would suggest the answer. Therefore, we can only assume. For example, assume that our hidden Multiverse is connected with other Multiverses which are unobservable not only by electromagnetic but also by gravitational manifestations. One of the probable structures of the hidden Multiverse is shown in Figure 3.

Let even more complex formations with a large number of the Multiverses including those with different structures (e.g., structure corresponding to the above-considered principle of physical reality of complex numbers) to be called a Hyperverse.

\subsection{Verification of the Structure of the Hidden Multiverse}

The above description of the structure of the hidden Multiverse raises a natural question whether this structure is verifiable. A large number of interesting Multiverse hypotheses, some of which are considered in [44]-[53], have been suggested so far. However, none of them is verifiable [54]. That is, they can be neither confirmed nor refuted by experiment even in the distant future.

The hypothesis of the hidden Multiverse is verifiable and therefore has every reason to be called a theory. Indeed, the theory of the hidden Multiverse:

- is confirmed experimentally by existence of dark matter and dark energy, which are 


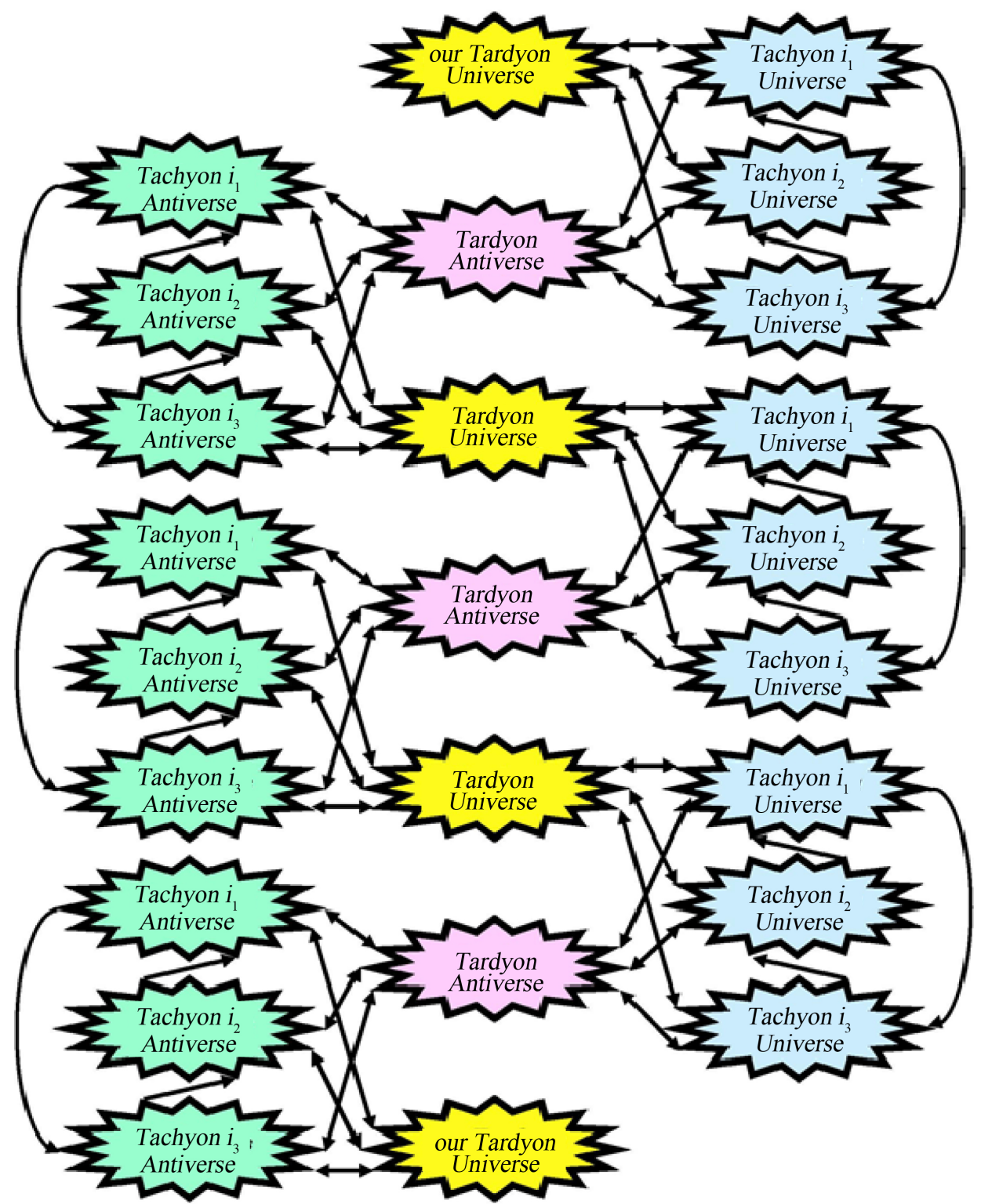

Figure 2. Probable structure of the hidden Multiverse corresponding to the principle of physical reality of quaternions.

just other names of invisible parallel universes of the hidden Multiverse, except our visible universe;

- is confirmed by those experiments at the Large Hadron Collider and other accelerators, which have resulted, explained by the formation of tachyons and their transition into tachyon universes and antiverses;

- can be confirmed by geophysical research of anomalous zones, proving that they are portals to adjacent parallel universes.

\section{Conclusions}

Thus, Ohm's law in the interpretation of Steinmetz has allowed simple and convincing 


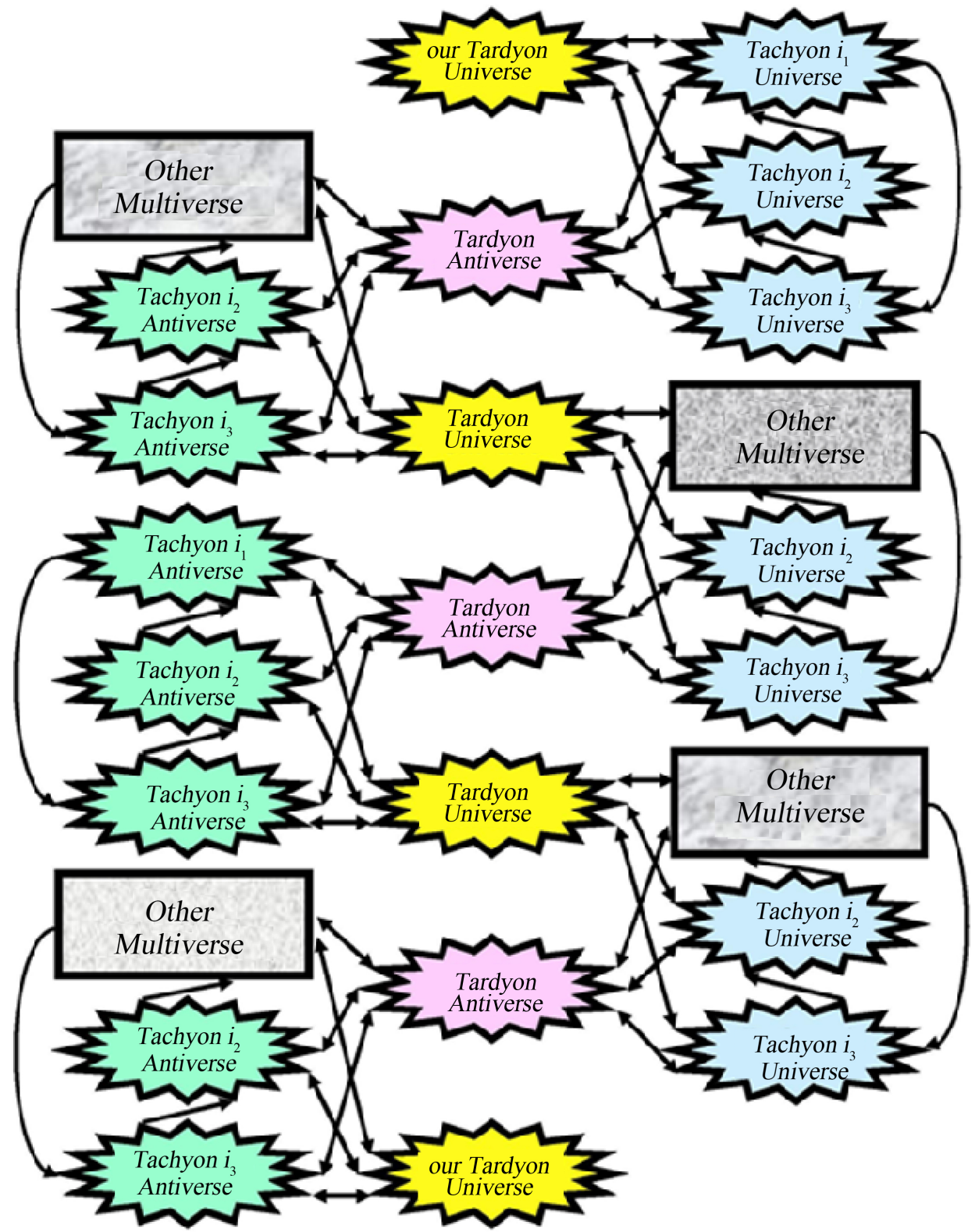

Figure 3. The option of an actual structure of the hidden Multiverse corresponding to the principle of physical reality of quaternions.

proving of physical reality of imaginary numbers, discovered five hundred years ago, in the process of study of oscillatory processes in linear electric circuits. This result, in contrast to the failed MINOS and OPERA physical experiments, can be repeated and verified in every electrical and electronic laboratory. Therefore, it is absolutely true and conclusive.

Since mathematics is the universal language of all exact sciences, the principle of physical reality of imaginary numbers is generally scientific. And all the theories and hypotheses in quantum mechanics, relativity theory, optics, geophysics, electronics and other sciences should be adjusted in accordance with this principle. 
Particularly in the STR this principle allowed identifying and correcting a number of fundamental errors that Albert Einstein had foreseen: "No single idea, which I would be sure that it will stand the test of time".

The adjusted version of the STR has, in turn, led to creation of the verifiable hypothesis of the hidden Multiverse, which is, therefore, has every reason to be called a theory, unlike other Multiverse hypotheses. Due to the WMAP and Planck data the theory allowed identifying the quaternion structure of the hidden Multiverse containing twenty to twenty-two parallel universes in the six-dimensional space. The theory has explained the phenomenon of dark matter and dark energy, which is just another name of invisible parallel universes of the hidden Multiverse, except ours. It also has explained what and where antimatter is, why it does not annihilate with matter and where tachyons can be found.

Consequently, Ohm's law in the interpretation of Steinmetz hasmade it possible to take the next step in the ideological revolution after the establishment of the Copernican heliocentric system, as it states that our universe is not the only one and is not the center of the hidden Multiverse. Moreover, our hidden Multiverse is as well not the only one, and it is not the center of the Hyperverse.

\section{Acknowledgements}

The author is grateful for participation in the discussion of the paper to my wife Dr. Olga Ilyinichna Antonova, whose criticism and valuable comments contributed to improvement of the article.

\section{References}

[1] Ohm, G.S. (1826) Journal für Chemie und Physik, 46, 137-166.

[2] Ohm, G.S. (1827) Diegalvanische Kette: Mathematisch Bearbeitet. Riemann, Berlin.

[3] Steinmetz, C.P. (2010) Theory and Calculation of Electric Circuit. Nabu Press, Charlstone.

[4] Weisstein, E.W., Ed. (2005) The CRC Concise Encyclopedia of Mathematics. 3rd Edition, CRS Press, Roca Raton.

[5] Einstein, A. (1920) Relativity: The Special and General Theory. H. Holt and Company, New York.

[6] Bohm, D. (2006) The Special Theory of Relativity. Routledge.

[7] Hawking, S.W. and Penrose, R. (2010) The Nature of Space and Time. Princeton University Press. https://doi.org/10.1515/9781400834747

[8] Antonov, A.A. (2014) Global Journal of Science Frontier Research: A Physics \& Space Science, 14, 51-59. https://doi.org/10.17686/sced rusnauka 2014-901

[9] Adamson, P., Ashby, N. and Bumgarner, R. (2007) Measurement of the Velocity of the Neutrino with MINOS. arXiv: 1408.6267v1 [physics.acc-ph].

[10] Adam, T., Agafonova, N., Aleksandrov, A., et al. (2011) Measurement of the Neutrino Velocity with the OPERA Detector in the CNGS Beam. arXiv: 1109.4897v4 [hep-ex].

[11] Antonello, M., Baibussinov, B., Boffelli, F., et al. (2012) Precision Measurement of the Neutrino Velocity with the ICARUS Detector in the CNGS Beam. arXiv:1208.2629 [hep-ex].

[12] Popper, K.R. (2002) Conjectures and Refutations: The Growth of Scientific Knowledge. Rout- 
ledge, London.

[13] Antonov, A.A. and Bazhev V.M. (1970) Means of Rising Deflecting Currents for Spiral Beam Sweep on the CRT Screen. Patent of USSR\#433650.

[14] Antonov, A.A. (2009) European Journal of Scientific Research, 28, 193-204. https://doi.org/10.17686/sced rusnauka 2009-885

[15] Antonov, A.A. (2010) General Mathematics Notes, 1, 11-16. https://doi.org/10.17686/sced rusnauka 2010-887

[16] Antonov, A.A. (2010) International Journal of Pure and Applied Sciences and Technology, 1, 1-12. https://doi.org/10.17686/sced rusnauka 2010-888

[17] Antonov, A.A. (2010) American Journal of Scientific and Industrial Research, 1, 342-349. https://doi.org/10.5251/ajsir.2010.1.2.342.349

[18] Antonov, A.A. (2015) General Mathematics Notes, 31, 34-53.

[19] Antonov, A.A. (2016) General Mathematics Notes, 35, 40-63.

[20] Einstein, A. (1905) Annalen der Physik, 17, 891-921. https://doi.org/10.1002/andp.19053221004

[21] Antonov, A.A. (2015) American Journal of Electrical and Electronics Engineeing, 3, 124129.

[22] Antonov, A.A. (2015) Global Journal of Physics, 2, 145-149.

[23] Antonov, A.A. (2016) Ponte, 72, 131-142. https://doi.org/10.21506/j.ponte.2016.7.9

[24] Frova, A. and Marenzana, M. (2006) Thus Spoke Galileo: The Great Scientist's Ideas and Their Relevance to the Present Day. Oxford University Press, New York.

[25] Tanaka, S. (1960) Progress of Theoretical Physics, 24, 171-200. https://doi.org/10.1143/PTP.24.171

[26] Feinberg, G. (1967) Physical Review, 155, 1089-1105. https://doi.org/10.1103/PhysRev.159.1089

[27] Antonov, A.A. (2014) American Journal of Scientific and Industrial Research, 5, 40-52.

[28] Antonov, A.A. (2015) International Journal of Advanced Research in Physical Science, 2, 25-32. https://doi.org/10.17686/sced rusnauka 2015-903

[29] Chernobrov, V.A. (2000) Encyclopedia of Mysterious Places of the Earth. Publishing House "Veche", Moscow.

[30] Freeman, K. and McNamara, G. (2006) In Search of Dark Matter. Springer, New York.

[31] Nicolson, I. (2007) Dark Side of the Universe: Dark Matter, Dark Energy, and the Fate of the Cosmos. Johns Hopkins University Press, Baltimore.

[32] Antonov, A.A. (2015) International Journal of Physics, 3, 84-87. https://doi.org/10.17686/sced rusnauka 2015-903

[33] Antonov, A.A. (2015) Global Journal of Science Frontier Research, 15, 33-38. https://doi.org/10.17686/sced rusnauka 2015-902

[34] Antonov, A.A. (2015) Cosmology, 19, 40-61. https://doi.org/10.17686/sced rusnauka 2015-903

[35] Antonov, A.A. (2015) Optics, 4, 43-47.

[36] Antonov, A.A. (2015) American Journal of Modern Physics, 4, 180-188. https://doi.org/10.11648/j.ajmp.20150404.14

[37] Antonov, A.A. (2016) Frontiers of Astronomy, Astrophysics and Cosmology, 2, 1-9.

[38] Antonov, A.A. (2016) Journal of Modern Physics, 7, 1228-1246. 
https://doi.org/10.4236/jmp.2016.710111

[39] Antonov, A.A. (2016) Ponte, 72, 288-300. https://doi.org/10.21506/j.ponte.2016.9.22

[40] Hinshaw, G., Larson, D., Komatsu, E., et al. (2013) Nine Year Wilkinson Anisotropy Probe (WMAP) Observations: Cosmological Parameter Results. arXiv: 1213.5226 [astro-ph/CO]

[41] Adam, R., Ade, P.A.R., Aghanim, N., et al. (2015) Plank 2015 Results. 1. Overview of Products and Scientific Results. arXiv:1502.01582v2 [astro-ph.CO]

[42] Korn, G.A. and Korn, T.M. (2000) Mathematical Handbook for Scientists and Engineers: Definitions, Theorems, and Formulas for Reference and Review. Courier Dover Publications, New York.

[43] Antonov, A.A. (2015) Global Journal of Science Frontier Research, 15, 8-15.

[44] Deutch, D. (1998) The Fabric of Reality: The Science of Parallel Universes and Its Implications. Penguin Books, New York.

[45] Greene, B. (2000) The Elegant Universe: Superstrings, Hidden Dimensions, and the Quest for the Ultimate Theory. Random House Inc., New York.

[46] Steinhardt, P.J. and Turok, N. (2007) Endless Universe: Beyond the Big Bang. Doubleday, New York.

[47] Vilenkin, A. (2006) Many Worlds in One: The Search for Other Universes. Hill and Wong, New York.

[48] Weinberg, S. (2008) Cosmology. Oxford University Press, Oxford.

[49] Carr, B. (Ed.) (2009) Universe or Multiverse? Cambridge University Press, Cambridge.

[50] Lucash, V.N. and Mikheyeva, E.V. (2010) Physical Cosmology. Physmathlit, Moscow.

[51] Greene, B. (2011) The Hidden Reality: Parallel Universes and the Deep Laws of the Cosmos. Random House Inc., New York.

[52] Deutsch, D. (2012) The Beginning of Infinity: Explanations That Transform the World. Reprint Edition, Penguin Books, New York.

[53] Tegmark, M. (2015) Our Mathematical Universe: My Quest for the Ultimate Nature of Reality. Vintage, New York.

[54] Antonov, A.A. (2016) Global Journal of Science Frontier Research, 16, 4-12.

\section{Submit or recommend next manuscript to SCIRP and we will provide best service for you:}

Accepting pre-submission inquiries through Email, Facebook, LinkedIn, Twitter, etc.

A wide selection of journals (inclusive of 9 subjects, more than 200 journals)

Providing 24-hour high-quality service

User-friendly online submission system

Fair and swift peer-review system

Efficient typesetting and proofreading procedure

Display of the result of downloads and visits, as well as the number of cited articles

Maximum dissemination of your research work

Submit your manuscript at: http://papersubmission.scirp.org/

Or contact jmp@scirp.org 